\title{
Téoros
}

Revue de recherche en tourisme

\section{Tremblant : la renaissance d'une destination de villégiature de calibre international...}

\author{
De Ryan à Intrawest... au-delà des moyens financiers
}

\section{Luc Tittley}

Volume 15, numéro 1, printemps 1996

Les Laurentides, quelles Laurentides?

URI : https://id.erudit.org/iderudit/1075051ar

DOI : https://doi.org/10.7202/1075051ar

Aller au sommaire du numéro

Éditeur(s)

Université du Québec à Montréal

ISSN

0712-8657 (imprimé)

1923-2705 (numérique)

Découvrir la revue

Citer cet article

Tittley, L. (1996). Tremblant : la renaissance d'une destination de villégiature de calibre international... De Ryan à Intrawest... au-delà des moyens financiers. Téoros, 15(1), 30-32. https://doi.org/10.7202/1075051ar 


\section{TREMBLANT: LA RENAISSANCE D'UNE DESTINATION DE VILLÉGIATURE DE CALIBRE INTERNATIONAL... \\ De Ryan à Intrawest... au-delà des moyens financiers.}

\section{Luc Tittley}

Luc Tittley
est géographe et urbaniste.
A titre de consultant
en planification et en
aménagement de produits et
de destinations touristiques,
il a été impliqué à plusieurs
reprises dans le dossier de la
station Mont-Tremblant et
d'autres stations touristiques
au Québec.

Qu plus connue des stations Louristiques du Québec, un géant endorml pendant une bonne vingtalne d'années, pulsse se révelller et reprendre ausst facllement sa position parml les plus grandes destinations quatre salsons d'Amérique du Nord ? L'envergure des réalisations et le rythme des transformations apportées récemment à la station créée en 1938 surprennent en cette période où l'économie est sl morose. Plusleurs Invoqueront, un peu rapidement, l'omnipulssance de l'argent pour expllquer le succès que connaît la station MontTremblant. Nous soutenons dans la réfexion qui suit que les capltaux n'expllquent pas tout. Ils accompagnent des projets blen pensés, lesquels s'appuient sur trols facteurs essentiels au succès : la vislon, le savolr-falre et l'appul du milleu.

\section{La vision}

Joseph Bondurant Ryan étalt un Américain entreprenant et fortuné de Philadelphle. Lors d'une mission d'exploration minlère, agrémentée d'un peu de chasse et de pêche, II avalt prolongé jusqu'à l'hiver son séjour à l'auberge de la famille Wheeler (le Grey Rocks Inn), tellement II appréclait la réglon de Salnt-Jovite, sa nature et ses gens. Nous sommes en 1937.

Au cours de ce même hiver, le Grey Rocks Inn reçolt la visite d'un dénommé Lowell Thomas, un skieur américain accompagné de son flls, qui propose à Joe Ryan l'ascenslon du mont Tremblant. Arrivé au sommet. Ryan fut sl Impressionne par le paysage spectaculaire de lacs et de montagnes modelées par les glaciers quí s'offralt à luI, qu'll résolut sur le champ de faire de ce site une destination de villégiature de premler choix.

Ryan. comme Thomas, avalt beaucoup voyagé et étalt féru de skl. Son objectif pour le mont Tremblant étalt simple: II voulait faire partager à ses amls américalns la beauté des paysages hlvernaux des Laurentides et de l'architecture paysanne du Québec. II voulait également offrir aux visiteurs une expérience unlque marquée par l'atmosphère chaleureuse et le dépaysement culturel des villages canadien-françals.

Les plans sont aussitôt dressés et, dès l"année sulvante, il mobilise la population locale pour entreprendre les travaux qul allalent mener à l'inauguration de la station en févirler 1939.

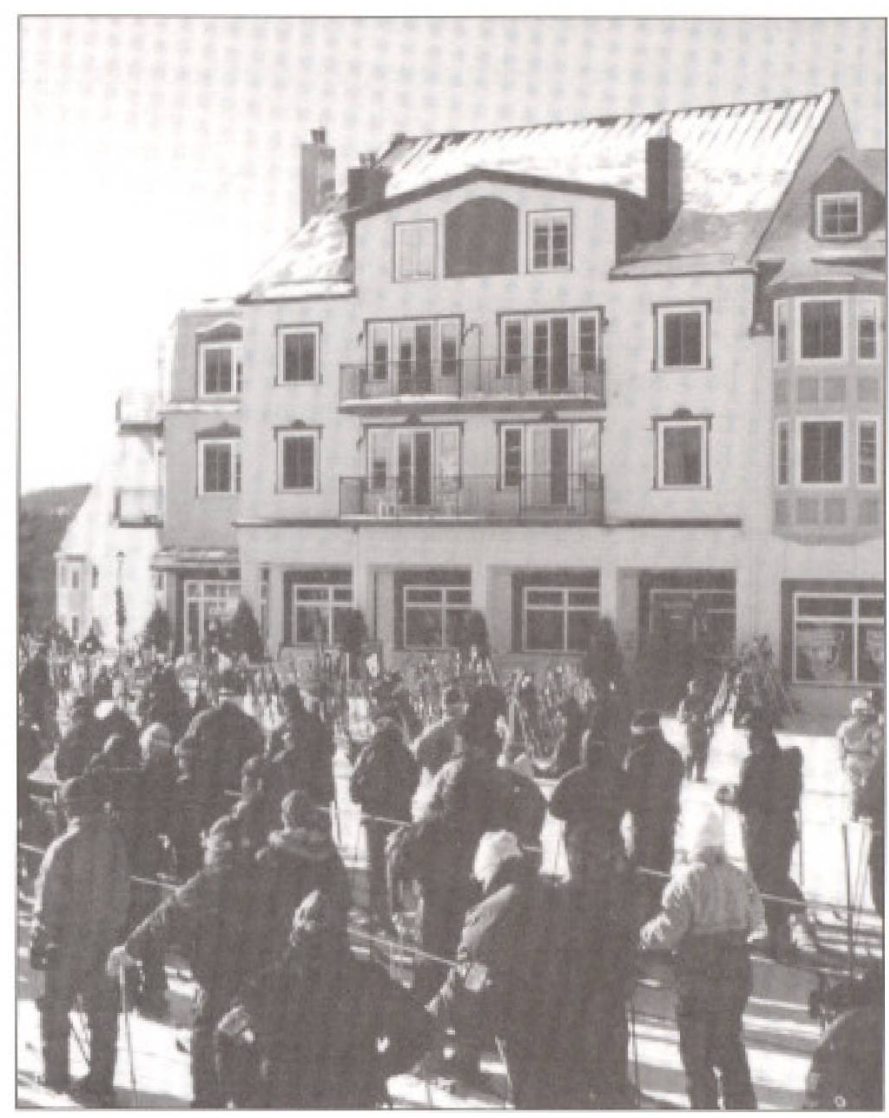

Le prodult avalt de la personnalité: un vllage québécols fait de malsons de bols badigeonnées à la chaux, avec son église. sa place, son auberge, quelques commerces et des chalets. Le succès fut immédlat auprès d'une cllentèle d'Américains biens nantis, amants des plaisirs de l'hiver assalsonnés d'un soupcon d'exotisme. Pourquol un tel succès?

- Le concept du produit étalt vendeur: les services et les equipements offerts correspondalent aux attentes de la clientèle.

- Le projet s'est développé selon un plan éclairé: les grandes orlentations du plan directeur de Joe Ryan (le vlllage et son emplacement, la configuration du domaine sklable. le partage entre les basses terres et les hautes terres, etc.) tiralent le meilleur part possible des attrlbuts du site.

- La commercialisation du prodult étalt bien clblée: on vlsalt avant tout une clientèle anglophone en quête d'aventure "douce".

- La réalisation ćtalt de quallté: le prodult llvré étalt à la hauteur des plans du promoteur et on n'avalt pas lésiné sur les moyens. 


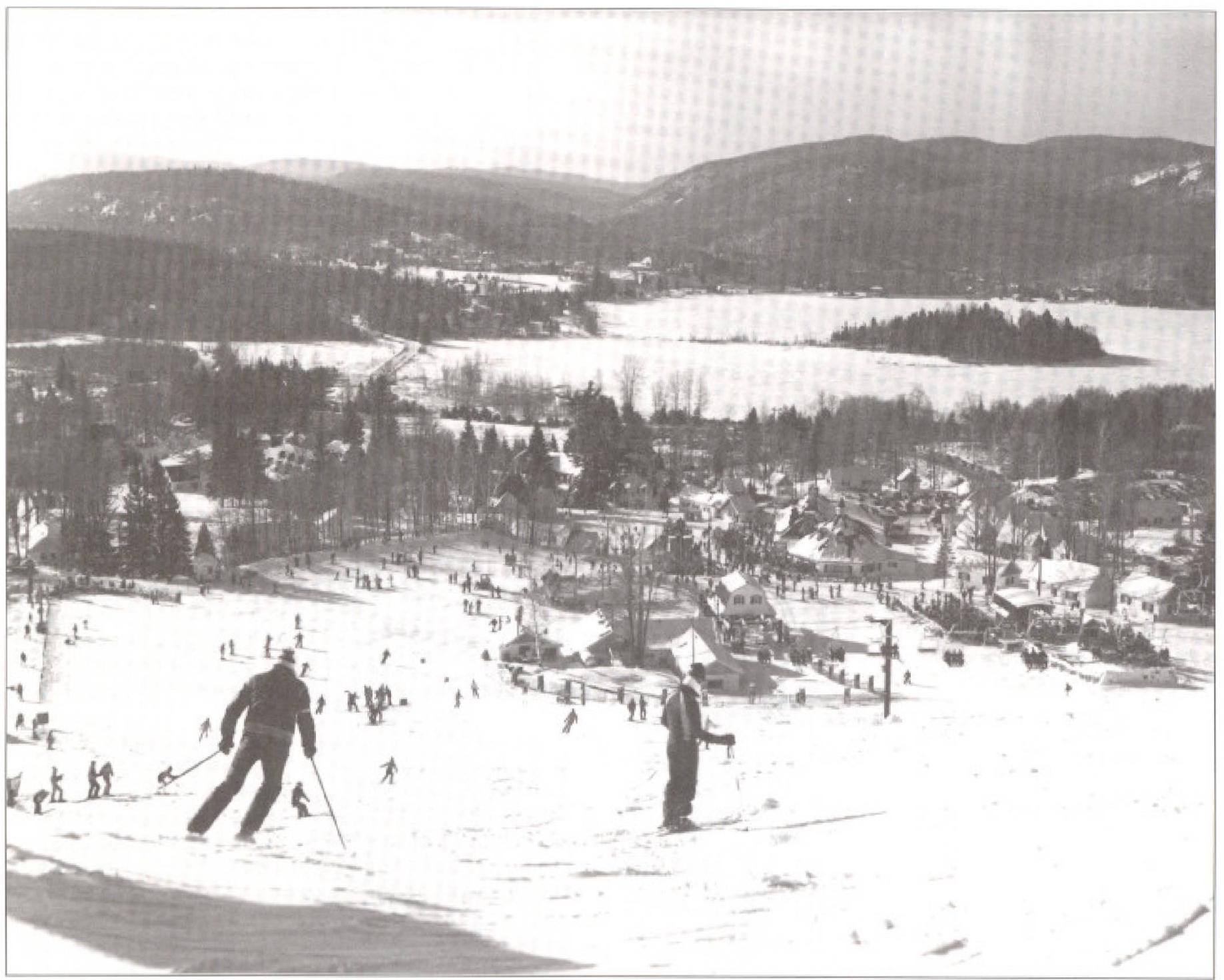

Le concept de base de Joe Ryan a survécu à tous les plans directeurs qui ont guidé l'évolution de la station depuis ce temps. Chacun des proprićtalres successifs a endossé la vislon stratéglque de Joe Ryan et s'est efforcé de préserver le caractère dlstinct du village,

Aujourd'hul, c'est au tour du groupe immobilier Intrawest de poursulvre la réalisation du rêve en respectant la vision initlale. Le défı est de talle, compte tenu des Impératifs économiques qui gouvernent la rentabllité des grandes statlons touristlques d'aujourd'hul: le besoln d'attelndre des niveaux élevés d'achalandage et la diversification des besoins et des attentes de la cllentèle touristique. Liaccueil généralement enthouslaste que reçolvent les réalisations actuelles portent à croire que le défl a été relevé.

\section{Le savoir-faire}

Les promoteurs touristiques enthouslastes, porteurs d'une vision inspirée. sont léglon. J'al pu le constater trop souvent. Ce qui manque le plus, c'est le savolr-falre... ces connaissances acquises (rarement infuses) qui permettent de concrétlser la vision.
Ce facteur de succès est complexe. II comporte plusieurs dimensions:

- Ie savolr-falre en matière de commerclalisation: la capacIté de concevolr et de réallser un marketing efficace auprès des bons marchés:

- Ie savoir-faire en matière d'exploitation: la capacité de gérer les actifs de façon efficiente et à la satisfaction de la clientèle:

- le savolr-faire en matlère de services à la clientèle: la capacité de recruter et de gérer avec succès un personnel enthousiaste et attentif.

La réussite vlent à ceux qui maîtrisent toutes ces dimensions. Le propriétaire actuel a montré beaucoup de maitrise dans ce domalne jusqu'à maintenant. Une visite de la station saura facilement en convaincre les plus sceptiques. Même les observateurs professionnels de l'Industrle des revues spécialisées ont répondu à la sollicitation du "géant qui se révellle" en reclassant Tremblant parml les grandes destinations de skl d'Amérique, statut que la station avalt perdu au cours de l'intérim chaotique entre l'époque de Joe Ryan et l'avènement d'Intrawest. 


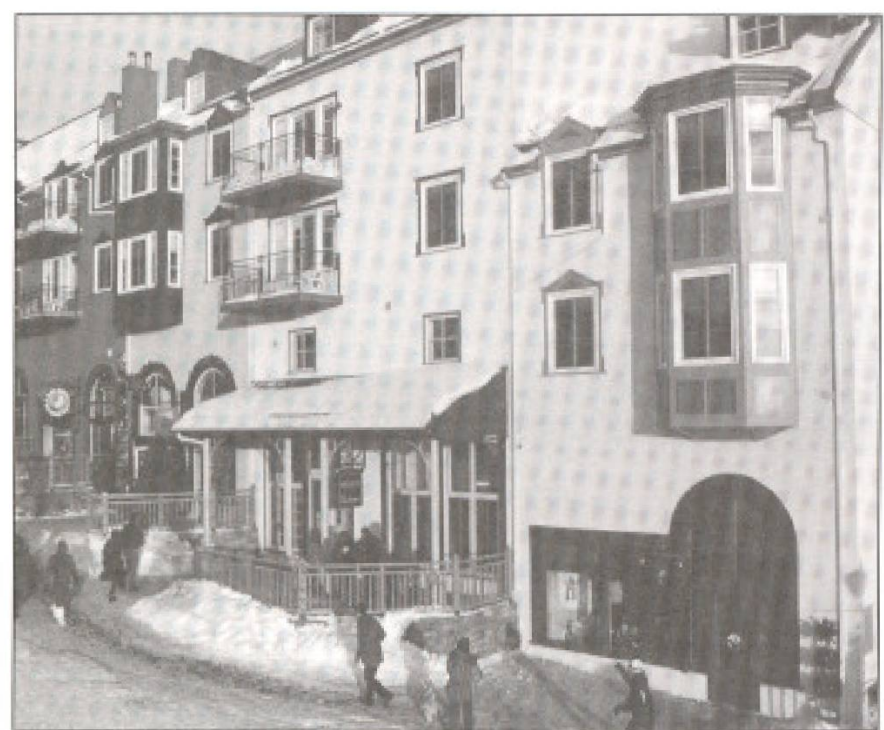

\section{L'appui du milieu}

Lappul accordé au promoteur par le milleu d'accuell joue une rôle essentiel dans le succès d'un projet touristique d'envergure. On pense Icl à l'appul des élus de tous les niveaux de gouvernement, des administrations publiques, du milleu financler, mals également à celul des intervenants privés de l'industrle tourlstlque de la région concernée.

Il est intéressant de noter que Joe Ryan fut reçu en sauveur en 1938, car il embauchalt les pères de famille du village de Lac Mercler (aujourd'hul, Municlpalité de Mont-Tremblant) pour débolser sa montagne et pour construlre son rêve. C'étalt encore la Dépression. On avalt aussı beaucoup bûché dans la région, sl bien que les petites entreprises locales vivant de la forêt fermalent par manque d'approvisionnement.

C'est un accuell semblable qu'on fit à Intrawest. Enfin, un exploitant digne de confiance après tant d'années de tiraillements, d'investissements mal planifiés et d'exploitation défclente. Aucun fantôme dans le placard de cet investisseur venu de l'Ouest, seule une crédibilité sans tache reposant sur la réussite de la station de Blackcomb à Whistler.

On attendait beaucoup et chacun a contribué sa part d'effort. Le succès obtenu jusquà date tient largement à ce que chacun des intervenants a blen joué son rỏle:

- le proprlétalre qul s'est empressé de définir un plan directeur ambiticux, offrant une combinaison de valeurs sûres et d'innovations, d'agir rapidement pour amélioner la qualité générale du produit et d'établir des liens avec le milleu;

- la municipallté qui mit de côté les querelles passées et étabilt une procédure moderne et un mécanisme sophistiqué de contrôle de l'utilisation du sol axé sur la protectlon de la qualité de l'environnement;

- les entreprises touristiques de la région, enfin affranchles de la dépendance trop longtemps entretenue à l'égard de la station, qui ont accepté le géant comme un partenatre à part entière et ont reconnu son rôle de moteur de l'économle réglonale:
- la fonction publique de tous les nlveaux de gouvernement qui n'a pas hésité à former, selon les besoins, des comités de travail mandatés pour agir rapidement:

- la main-d'oeuvre de la réglon quul a souscrit avec enthouslasme à la philosophie de gestion du nouveau propriétaire, laquelle constitue un élément essentlel du produit offert.

Le soutien au projet n'a pas été servile. Il est né dans l'enthouslasme, certes, mais Il s'est consolidé progressivement à la lumlère des gestes quotidlens. Ces gestes posés par les uns et par les autres ont falt la preuve que la conflance pouvait être réclproque entre le promoteur et le milleu d'accuell.

\section{Le passage de la tornade}

Certains intervenants touristiques de la région s'Inqulètent. non pas tant du succès de la station Mont-Tremblant, mals plutôt de la concentration des Investissements et de l'achalandage dans un même lleu. On croit assister au passage d'une tornade qul aspire toute l'économle touristique sur son passage.

Face à ce phénomène, II Importe de ne pas perdre de vue, dans la conjoncture actuelle, que ce n'est pas le ralentissement généralisé des investlssements allleurs qui est anormal, mals blen plutôt le falt que plus de 450 millions \$ d'argent essentilellement privé solent Investls à Tremblant.

Aussi, nous apparait-il plus utile pour l'Industrle touristique de la réglon de tabler sur l'effet d'entraînement du projet en termes de positionnement marketing. Ceux qut broyent du noir devralent plutôt se consacrer à améllorer ou à développer des prodults complémentalres susceptibles de consollder et de diversiffer l'offre de la réglon... car la station MontTremblant ne peut pas être tout pour tous.

En terminant, on me permettra de soulever deux défls à relever afin d'optimiser les retombées touristıques découlant du révell du géant:

- le malllage de la station Mont-Tremblant avec le Parc provincial du Mont-Tremblant. La ressource est là, mals l'intégration est falble. Loccasion est bonne de s'interroger sur les enjeux et les modalités de collaboration au moment où l'administration du parc est à revoir son plan directeur;

- et, comme corollaire à l'enjeu précédent, l'élargissement de la zone d'Influence au secteur de Salnt-Donat. Ce secteur, riche en ressources complémentalres, est actuellement Isolé de la station par une réseau routier davantage conçu pour perpétuer les tiralllements Laurentides-Lanaudlère que pour favorlser le renforcement du pôle touristıque le plus dynamique du Québec.

Icl comme allleurs, pour que le succès solt au rendez-vous. vision, savoir-faire et appui du millieu devront être à l'ordre du jour.

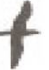

